\title{
Ward Round - A patient with multi-organ failure
}

\author{
A Stevenson, C Phiri, J Mallewa, M Molyneux \\ 1.Department of Surgery, College of Medicine, University of Malawi, \\ Blantyre \\ 2.Department of Paediatrics, Queen Elizabeth Central Hospital, Blantyre
}

A 33 year old woman was admitted to the gynaecology ward in Queen Elizabeth Central Hospital with a four day history of abdominal pain and jaundice. She was 16 weeks pregnant in her third pregnancy. Both her previous pregnancies resulted in live, healthy children. Her health passport showed that she had been diagnosed HIV positive in 2008 during antenatal testing, although neither the patient nor her relatives were aware of her status and she was not taking anti-retroviral treatment. Three weeks before admission she had received treatment for malaria with quinine and had made a full recovery. She had no other past medical history.

On admission she was jaundiced and looked unwell. She had oral candidiasis. There was no wasting, lymphadenopathy or signs of chronic liver disase. She was apyrexial, her blood pressure was $89 / 53$, her respiratory rate was 40 breaths per minute with oxygen saturation $96 \%$ when breathing air. Her liver was tender and palpable $3 \mathrm{~cm}$ below the costal margin. She had a clear chest and no other abnormal findings. She was alert and fully orientated.

Later on the day of admission she developed a fever and was started on intravenous ceftriaxone $2 \mathrm{~g}$ once a day. Her blood tests on admission are shown in the Table 1 below.

On review the next day she was noted to be in respiratory distress with a respiratory rate of 52 breaths per minute and oxygen saturation $85 \%$ on air. Her blood pressure was $100 / 60$ with a heart rate of 140 beats per minute. Her chest was clear. Her saturations improved to $97 \%$ on 4 litres/ minute of oxygen.

On the third day of admission the patient had a spontaneous miscarriage, and a uterine evacuation was performed under paracervical block. Two days later she became unconscious with a Glasgow Coma Score of 5/15. She had equal pupils, no neck stiffness and no focal abnormality. No seizure activity had been witnessed.

Further blood tests showed a raised serum creatinine concentration (see Table 1b). Her urine output was adequate throughout her admission. Urinalysis showed haematuria but no proteinuria. An abdominal ultrasound showed a normal liver, normal calibre bile ducts and swollen kidneys. An EEG showed diffuse generalized widening and slowing of waves, a pattern consistent with a systemic metabolic encephalopathy. [See Figure 1a. A normal EEG is shown in Fig $1 \mathrm{~b}$ for comparison].

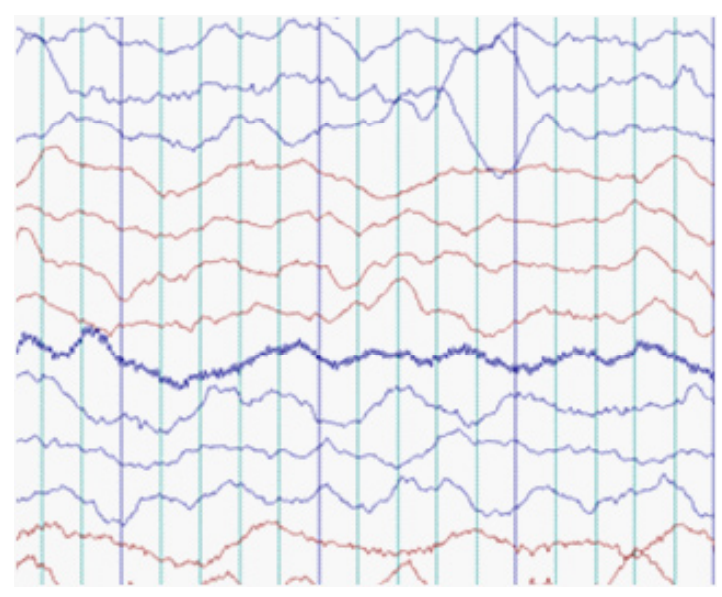

Fig la. Part of the patient's EEG on day 6

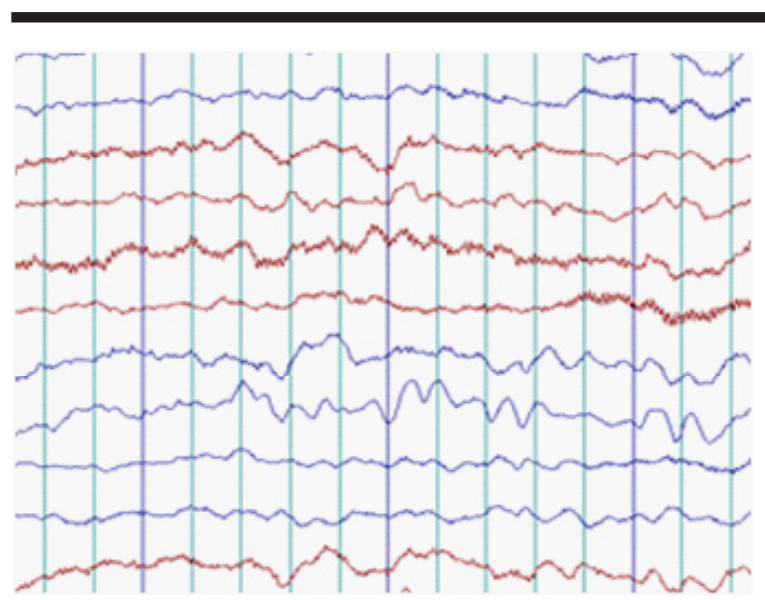

\section{Fig 1b. Part of a normal EEG (for comparison)}

At this point, all her medications were stopped and she was given liquid paraffin to prevent constipation worsening her encephalopathy. Her coma score improved slowly over the following $48 \mathrm{~h}$ hours and by day seven of admission she could respond to speech, although she remained drowsy.

Over the next week her jaundice resolved completely, her renal function improved and her level of consciousness returned to normal.

On day 14 of admission she complained of being unable to walk due to weakness and a sense of heaviness in both legs. On formal examination there was mild proximal weakness in her legs with normal sensation in all modalities. A neurologist assessed the patient and suggested that the weakness was due to a myopathy rather than neurological problem.

Her leg weakness improved spontaneously, and four weeks after admission she was alert and fully ambulant and was discharged from hospital. 


\begin{tabular}{|l|l|l|l|l|}
\hline & Day 1 & Day 4 & Day 7 & $\begin{array}{l}\text { (Normal } \\
\text { ranges) }\end{array}$ \\
\hline Haemoglobin & $8.9 \mathrm{~g} / \mathrm{dL}$ & & & $(11-16)$ \\
\hline MCV & $86 \mathrm{fl}$ & & & $(76-96)$ \\
\hline Platelets & $71103 / \mathrm{mm} 3$ & & & $(150-400)$ \\
\hline White cells & $9103 / \mathrm{mm} 3$ & & & $(4-11)$ \\
\hline CD4 count & 22 cells/ mm3 & & & \\
\hline $\begin{array}{l}\text { Thick film } \\
\text { for malaria }\end{array}$ & Negative & & & \\
\hline AST & $246 \mathrm{U} / \mathrm{L}$ & $67 \mathrm{U} / \mathrm{L}$ & & $(0-50)$ \\
\hline ALT & $156 \mathrm{U} / \mathrm{L}$ & $13 \mathrm{U} / \mathrm{L}$ & & $(0-50)$ \\
\hline $\begin{array}{l}\text { Alkaline } \\
\text { phosphatase }\end{array}$ & & $226 \mathrm{U} / \mathrm{L}$ & & $(100-290)$ \\
\hline $\begin{array}{l}\text { Bilirubin } \\
\text { (direct) }\end{array}$ & $3.7 \mathrm{mg} / \mathrm{dL}$ & $10 \mathrm{mg} / \mathrm{dL}$ & & $(0.2-0.5)$ \\
\hline $\begin{array}{l}\text { Bilirubin } \\
\text { (total) }\end{array}$ & $5.0 \mathrm{mg} / \mathrm{dL}$ & $14 \mathrm{mg} / \mathrm{dL}$ & & $(0.4-1.0)$ \\
\hline Creatinine & & $7.4 \mathrm{mg} / \mathrm{dL}$ & 2.13 & $(0.7-1.4)$ \\
\hline $\begin{array}{l}\text { Blood } \\
\text { urea } \\
\text { nitrogen }\end{array}$ & & $9 \mathrm{mg} / \mathrm{dL}$ & & $(5-60)$ \\
\hline
\end{tabular}

Table 1: Patients blood tests results

miscarriage.

A possible agent is Vernonia amygdalina Del. (Asteraceae). This is a medicinal plant found in tropical Africa which is sometimes used as an anti-malarial treatment ${ }^{2,3}$. It has also been used in Malawi as an oxytocic agent to help induce uterine contractions and potentially to induce abortion ${ }^{4}$. Our patient may have taken this remedy for her recent illness with suspected malaria, accidentally resulting in miscarriage.

This was an unusual case of multi-organ failure in early pregnancy with a rapid, spontaneous recovery. Although we have suggested several possible causes, a unifying diagnosis has remained elusive.

\section{References}

1.Burrow, G., Duffy, T. (1999). Medical Complications During Pregnancy Philadelphia: W.B. Saunders Company

2.Erasto, P., Grierson, D., Afolayan, A., (2007) Evaluation of antioxidant activity and the fatty acid profile of the leaves of Vernonia amygdalina growing in South Africa Food Chemistry 104: 636-642

3.Tona, L., Cimanga, R., Mesia, K., et al. (2004) In vitro antiplasmodic activity of extracts and fractions from seven medicinal plants used in the Democratic Republic of Congo. J Ethnopharmacol. 93: 27 - 32

4.Bullough, C.H.W., Leary, W.P. (1982). Herbal Medicines used by Traditional Birth Attendants in Malawi. Trop Geograph. Med 34: 8185

What might have caused this patient's illness?

\section{Discussion of Case.}

We postulated several possible causes for this patient's presentation with jaundice, encephalopathy, spontaneous miscarriage, acute renal failure and proximal myopathy. Liver disease was the main feature of this case, and although there are several well known causes of liver disease in pregnancy, none seemed particularly likely in our scenario.

Acute fatty liver of pregnancy can cause progressive jaundice and encephalopathy, and 50\% of cases are complicated by acute renal failure. It is a rare condition, which tends to occur later in pregnancy ${ }^{1}$. Diagnosis is clinical. Ultrasonography shows a 'normal' liver as the fat deposits are microvesicular. Treatment is delivery of the fetus, with liver function tests rapidly returning to normal and full recovery in most cases. Recurrence in subsequent pregnancies is rare. This diagnosis would not explain why our patient deteriorated after her miscarriage, unless a second disease process occurred simultaneously.

We considered a drug-induced hepatitis. However the patient had not taken any medications prior to admission, and her jaundice developed before she started to receive ceftriaxone (a recognised cause of jaundice). Neither was she taking anti-retroviral therapy, a well known cause of hepatotoxicity. Quinine can cause acute renal failure but is not hepatotoxic, and is generally considered safe for use in pregnancy at therapeutic doses.

The spontaneous and rapid resolution of her symptoms prompted us to consider toxin ingestion as a cause. Patients in Malawi may use local herbs and traditional remedies prior to seeking conventional medical treatment. Our patient may have taken a local remedy, with the unintended side effects of hepatoxicity and miscarriage. Alternatively she may have taken a traditional remedy to deliberately induce 